\title{
Abnormal QT Interval Variability in Patients with Multiple System Atrophy
}

\author{
S Cuomo $^{1}$, MA Ribani ${ }^{2}$, F Marciano ${ }^{3}$, ML Migaux ${ }^{3}$, P Cortelli $^{4}$, \\ G Pierangeli ${ }^{4}$, PE Russo $^{1}$, A D'Andrea ${ }^{1}$, R Calabrò ${ }^{1}$ \\ ${ }^{1}$ Cardiologia SUN, AO Monaldi Napoli, Italy \\ ${ }^{2}$ Cardiologia, AO Belluria, Bologna, Italy \\ ${ }^{3}$ Istituto di Cibernetica CNR, Pozzuoli, Italy \\ ${ }^{4}$ Dipartimento Scienze Neurologiche,Università di Bologna, Italy
}

\begin{abstract}
In a prospective study, we evaluated the hypothesis that QT variability is abnormal in multiple system atrophy. Time and frequency domain $Q T$ variability was assessed from 24-hour Holter monitoring Three-channel Holter monitoring was performed in all subjects and was analyzed on a homemade analyzer. The 24-hour mean QT duration was similar in multiple system atrophy patients and in control subjects Unlike $Q T$ duration, several parameters of time and frequency domain $Q T$ variability were significantly increased in multiple system atrophy patients compared to healthy controls. Time domain measures of QT variability were significantly shorter while asleep than while awake in both groups. The nighttime \% reduction in time-domain $Q T$ variability tended to be smaller in patients in comparison to control subjects, but this difference did not achieve statistical significance This is the first reporting demonstrating that $Q T$ variability is abnormal in patients with multiple system atrophy.
\end{abstract}

\section{Introduction}

Multiple system atrophy (MSA) is an adult-onset neurodegenerative disorder, showing various combination of progressive autonomic failure, cerebral ataxia, and levodopa poorly responsive parkinsonism [1]. An important clue to the diagnosis of multiple-system atrophy is the recognition of several early autonomic symptoms: fatigue, weakness, orthostatic dizziness, decreased sweating, discoloration of the hand or foot (or both) on one side, erectile dysfunction (in men), bladder dysfunction, changes in bowel motility, and sleep abnormalities [2]. Autonomic nervous system tone has been evaluated extensively in humans by employing the tool of heart rate variability, which relies on the principle that the pattern of beat-to-beat control of the sinoatrial node provides a reflection of autonomic activity. More recently, QT variability, a measure of the cyclic variations of beat-to-beat QT intervals, has been proposed as new approach to the assessment of cardiac autonomic function. QT variability can be assessed using a number of algorithms currently in various phases of development and validation. Increased QT variability has been described in several conditions with increased risk of arrhythmias and sudden death [ 3, 4 ], so that quantitative measurement of QT variability is considered to provide a non-invasive measure of susceptibility to ventricular arrhythmias[5]. However, to our knowledge, attempts to analyze QT variability have never been performed on 24hour Holter recordings in patients with MSA, although these patients appear to be at higher risk of lifethreatening arrhythmias, due to autonomic dysfunction. Therefore, we measured QT variability in a small group of patients with MSA. Our hypothesis was that QT variability was greater in this group of patients than in normal subjects.

\section{Methods}

Seven consecutive patients with MSA, 4 males and 3 females, mean age $66 \pm 12$ years, and 7 healthy subjects, 4 females and 3 males, mean age $54 \pm 11$ years were included in the study. All the patients had clinical characteristics which correspond to a probable MSA diagnosis [1]. Healthy subjects had negative physical examinations and normal electrocardiograms. All study subjects were ambulatory and had a 24-hour Holter monitoring, showing sinus rhythm, normal QRS duration and clear end point of the $\mathrm{T}$ wave in at least one lead. None of the study patients received antiarrhythmic drugs at the time of the Holter recordings. All the study subjects were asked to maintain their normal activities and their 
normal asleep-awake rhythm during the recording.

Three-channel 24-hour Holter monitoring was performed in all subjects with a Del Mar Avionics (Irvine, California,USA) model 459 recorder. Holter recordings were analyzed on a homemade analyser built around a Motorola 68030-50 Mhz microprocessor ( Motorola Inc. Libertyville, IL, USA). Operating characteristics of this analyser have been previously reported [6]. Briefly, 3 recorded ECG analog channels were read via a modified Teac-Tascam 234 Syncaset tape deck ( Teac Co., Tokyo, Japan) and digitised at 200 samples/sec. Besides evaluation of the usual ECG parameters, including identification of QRS widths and shapes and of RR interval abnormalities, QT intervals at each beat were evaluated as follows. The interval between the peak of the $R$ wave $\left(R_{a}\right)$ and the end of the $T$ wave $\left(\mathrm{T}_{\mathrm{e}}\right)$ was found in a 2 steps process.

Table 1 Frequency Domain Measures

\begin{tabular}{|l|c|}
\hline Variable & Area under the power spectrum in the range \\
\hline Total Power $\left(\mathrm{ms}^{2}\right)$ & 0.00066 to $0.34 \mathrm{~Hz}$ \\
\hline Ultra low frequency power $\left(\mathrm{ms}^{2}\right)$ & 0.00066 to $0.00033 \mathrm{~Hz}$ \\
\hline Very low frequency power $\left(\mathrm{ms}^{2}\right)$ & 0.0033 to $0.04 \mathrm{~Hz}$ \\
\hline Low frequency power $\left(\mathrm{ms}^{2}\right)$ & 0.04 to $0.15 \mathrm{~Hz}$ \\
\hline High frequency power $\left(\mathrm{ms}^{2}\right)$ & 0.15 to $0.34 \mathrm{~Hz}$ \\
\hline
\end{tabular}

Table 2. Definition of Time Domain Variables of QT Interval Variability

\begin{tabular}{|l|l|}
\hline Variable & Definition \\
\hline SDNN (ms) & Standard deviation from the mean of all NN QT intervals of sinus beats over 24 hours \\
\hline SDANN $(\mathrm{ms})$ & $\begin{array}{l}\text { Standard deviation of the averaged NN QT intervals of sinus beats for all 5-min } \\
\text { Segments }\end{array}$ \\
\hline SDNN index (ms) & $\begin{array}{l}\text { Mean of the standard deviations of all NN QT intervals of sinus beats for all 5-minute } \\
\text { segments of a 24-hour ECG recording }\end{array}$ \\
\hline rMSSD (ms) & $\begin{array}{l}\text { The square root of the mean of the squared differences between adjacent NN QT } \\
\text { intervals of sinus beats over the entire 24-hour ECG recording }\end{array}$ \\
\hline pNN50 $(\%)$ & $\begin{array}{l}\text { Percentage of adjacent QT intervals differing by more than } 50 \text { ms, computed over the entire } \\
\text { 24-hour ECG recording }\end{array}$ \\
\hline
\end{tabular}

Firstly, a table relating the values of the intervals between $R_{a}$ and the peak of the $T$ wave $T_{a}$ to $R R$ intervals was built into the program. The $\mathrm{T}_{\mathrm{a}}$ point was then searched within a moving window centered around the value given by the table. Then the interval $T_{a} T_{e}$ was measured. The maximum slope value was found computing slopes after $\mathrm{T}_{\mathrm{a}}$ as moving averages of the slopes measured at four consecutive sample points, reducing in so doing the influence of artifacts and noise. It was, then, possible to derive a threshold as a fraction of this maximum value. Several consecutive slopes after the maximum were then compared with this threshold.

The first point where the slope was smaller then the threshold was taken as $T_{e}$. Finally $Q R_{a}$ value is added to $\mathrm{R}_{\mathrm{a}} \mathrm{T}_{\mathrm{e}}$ to form the QT interval. The sequences of all RR and QT intervals were stored with a code number identifying its normality or their class of abnormality. Premature ventricular complexes and their adjacent beats, used only for time keeping purposes, were rejected just as noise and other aberrant ECG signals. Data losses did not exceed $15 \%$ of the total recordings.

The sequences of all normal RR (NN) and their corresponding QT intervals were analysed in order to compute time and frequency domain measures of QT variability for the entire $24 \mathrm{~h}$ recordings as well as for awake and sleep periods.

The $24 \mathrm{~h}$ power spectrum estimates were computed by means of the fast Fourier transforms and by averaging a sufficient number of spectra so as to reduce the estimation error. Time functions samples were obtained from the QT 
sequences by linear interpolation with time steps of 100 ms; this is a low-pass filtering operation which attenuates any variability above the chosen value of the sampling frequency. The frequency bands explored are reported in
Table 1. The following time domain parameters of QT variability were calculated: SDNN, SDNN index, SDANN, rMSSD, pNN50. The definition and statistical procedures of these parameters are listed in Table 2.

Table 3. Time Domain QT Variability in MSA Patients and Control Subjects

\begin{tabular}{|l|l|l|l|}
\hline \multicolumn{1}{|c|}{ Variable } & MSA Patients & Control Subjets & $\mathrm{p}$ \\
\hline 24-hour QT-SDNN (ms) & $82 \pm 3$ & $47 \pm 24$ & .003 \\
\hline 24-hour QT-SDANN (ms) & $14 \pm 5$ & $21 \pm 9$ & .104 \\
\hline 24-hour QT-SDNN index (ms) & $23 \pm 7$ & $16 \pm 3$ & .035 \\
\hline 24-hour QT-rMSSD (ms) & $33 \pm 9$ & $20 \pm 4$ & .005 \\
\hline 24-hour QT-pNN50(\%) & $7.4 \pm 5.7$ & $3.8 \pm 2.9$ & .165 \\
\hline
\end{tabular}

Table 4. Frequency Domain QT Variability in MSA Patients and Control Subjects

\begin{tabular}{|l|l|l|l|}
\hline \multicolumn{1}{|c|}{ Variable } & MSA Patients & Control Subjets & $\mathrm{p}$ \\
\hline 24-hour QT total power & $7.1 \pm .3$ & $7.0 \pm .2$ & .329 \\
\hline 24-hour QT ultra low frequency power & $6.3 \pm .4$ & $6.4 \pm .3$ & .523 \\
\hline 24-hour QT very low frequency power & $5.6 \pm .3$ & $5.2 \pm .2$ & .034 \\
\hline 24-hour QT low frequency power & $5.3 \pm .3$ & $5.0 \pm .3$ & .033 \\
\hline 24-hour QT high frequency power & $5.2 \pm .3$ & $4.9 \pm .2$ & .041 \\
\hline
\end{tabular}

\section{Results}

The 24-hour mean QT duration was similar in multiple system atrophy patients and in control subjects $(373 \pm 19$ vs $382 \pm 20 . p=.406$ ). Summary data on time domain QT variability, comparing MSA patients with control subjects, are tabulated in Table 3. Unlike QT duration, several parameters of time domain QT variability were significantly increased in MSA patients compared to healthy controls (SD $82 \pm 3$ vs $47 \pm 24 \mathrm{p}=.003$; SDIND $23 \pm 7$ vs $16 \pm 3 \mathrm{p}=.035$; $\mathrm{r}-\mathrm{MSSD} 33 \pm 9$ vs $20 \pm 4, \mathrm{p}=.005$ ) (Table 3). When a frequency domain analysis was used, there was no significant difference between MSA patients and healthy controls, however several measures of frequency domain QT variability tended to be higher in patients with MSA than in control subjects (Very Low Frequency Power $\ln 5.6 \pm 0.3$ vs $5.2 \pm 0.2 \mathrm{p}=.034$; Low Frequency Power $\ln 5.3 \pm 0.3$ vs $5 \pm 0.3 \mathrm{p}=.033$; High Frequency Power $\ln 5.2 \pm 0.3$ vs $4.9 \pm 0.2 \mathrm{p}=.041$ ) (Table 4). Time domain measures of QT variability were significantly shorter while asleep than while awake in both groups. The night-time $\%$ reduction in time-domain QTV tended to be smaller in patients in comparison to control subjects, but this difference did not achieve statistical significance (mean $\%$ reduction in time domain parameters $-26 \pm 19$ vs $-36 \pm 19 \%, \mathrm{p}=0.296$ ).

\section{Discussion and conclusions}

We believe this is the first report examining the QT variability in MSA. Several methods have been proposed to quantify abnormalities of ventricular repolarization. However, few techniques are suitable for routine clinical use. Thus, assessment of ventricular repolarization still is based largely on QT, QTc and QT dispersion measurements. We analyzed beat-by-beat QT variability using an home-developed program. Our results suggest that QT variability is greater in MSA patients compared with healthy controls. Moreover, a trend toward smaller changes in QT variability between daytime and nighttime was evident in MSA patients in comparison to healthy controls. This finding resembles the absence of circadian change in heart rate variability in patients with increased risk of death after myocardial infarction [ 7, 8 ]. Changes in autonomic tone in MSA may explain both increased 
QT variability as well as a trend toward reduced daynight differences in time domain QT variability and may represent a predisposing condition to arrhythmogenesis. A relationship between increased QT variability and enhanced risk of life-threatening arrhythmias has been previously demonstrated in several conditions with increased risk of arrhythmias and sudden death [ 4, 5 ]. Thus, we hypothesize that in MSA temporal liability in ventricular repolarization might be mechanistically related to increased risk of malignant ventricular arrhythmias and sudden death. We perceive that our study is hypothesis-generating, and not aimed at identifying associations between abnormal QT variability and prognosis. The increase in QT variability found in our case-control study does not allow us to establish a mechanistic link between abnormal QT variability and sudden death in MSA patients. Therefore, this novel association should be investigated in future studies. Confirming abnormal QT variability as a novel risk factor for sudden death in MSA might open new perspectives for the selection of the appropriate preventive management.

A major limitation of the present study should be pointed out. The number of patients enrolled was too small to draw definite conclusions. Inadequate power due to insufficient sample size might explain why no significant differences were observed in several QT variability parameters between MSA patients and healthy subjects.

In conclusion, this is the first prospective study to identify an association between abnormal QT variability and MSA. The prognostic value of this finding remains unknown. Thus, these results should stimulate further study to evaluate if the estimation of QT variability by ambulatory ECG monitoring offers prognostic information beyond that provided by the evaluation of current employed clinical variables.

\section{References}

[1] Gilman S, Low PA, Quinn N, et al. Consensus statement on the diagnosis of multiple system atrophy. J Neurol Sci 1999;163:94-98

[2] Schlossmacher MG, Hamann C, Cole AG, Gonzalez G,Frosch MP. Case 27-2004: a 79year-old woman with disturbances in gait, cognition and autonomic function. $\mathrm{N}$ Engl $\mathrm{J}$ Med 2004;351:912-22

[3] Atiga WL, Calkins H, Lawrence JH, Tomaselli GF, Smith JM, Berger RD Beat-to-beat repolarization lability identifies patients at risk for sudden cardiac death. J Cardiovasc Electrophysiol 1998;9:899-908

[4] Haigney MC, Zareba W, Gentlesk PJ, Goldstein R, Illovsky M, McNitt S, Andrews ML, Moss AJ. QT interval variability and spontaneous ventricular tachycardia or fibrillation in the Multicenter Automatic Defibrillator Implantation Trial (MADIT) II patients. J Am Coll Cardiol 2004;44:1481-7.

[5] Berger RD. QT variability. J Electrocardiol 2003; 36 (Suppl. 2003): 83-87

[6] Marciano F, Cuomo S, Migaux ML, Boccalatte M, Betocchi S. Classification of Patients with and without Syncope by means of QT Analysis in Hypertrophic Cardiomiopathy : Preliminary results. Computers in Cardiology 2001;28;381383

[7] Itou T, Obata S, Tateishi O. Characteristics of the circadian rhythm of heart rate variability in patients with sudden cardiac death after myocardial infarction. A.N.E. 1998; 3:183.

[8] Malik M, Farrell T, Camm AJ. Circadian rhythm of heart rate variability after acute myocardial infarction and its influence on the prognostic value of heart rate variability. Am J Cardiol 1990; 66:1049

Address for correspondence

F. Marciano

Istituto di Cibernetica del CNR

Via Campi Flegrei 34

80078 Pozzuoli, Napoli, Italy

formar@biocib.cib.na.cnr.it 